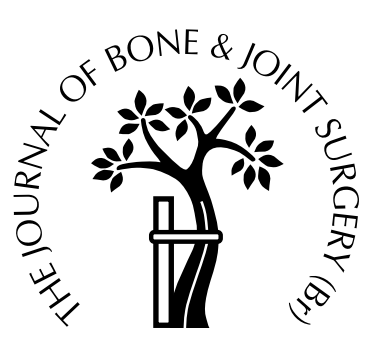

\title{
Estimation of the extent of osteonecrosis of the femoral head using MRI
}

\author{
Young-Min Kim, Jae Hoon Ahn, Heung Sik Kang, Hee Joong Kim \\ From Seoul National University Hospital, Seoul, Republic of Korea
}

$\mathbf{T}$ The extent of necrosis is the main determining factor in the outcome of osteonecrosis of the femoral head and is best measured by MRI.

Using statistical analyses, we have evaluated the accuracy and repeatability of seven different methods of measuring the extent of necrosis, of which three demonstrate good or acceptable accuracy. For the general observer their repeatability is poor; only for experienced observers were they reasonable. We conclude that within an institution or for personal use, the accuracy of some methods is acceptable, and repeatability reasonable. None is appropriate for international use.

J Bone Joint Surg [Br] 1998;80-B:954-8.

Received 26 August 1997; Accepted after revision 16 February 1998

It is generally accepted that in osteonecrosis of the femoral head $(\mathrm{ONFH})$, the size of the necrotic segment correlates closely with both the natural history and the results of treatment. ${ }^{1-8}$ MRI allows accurate evaluation of ONFH and most authors now use this technique to estimate the size of a necrotic lesion. Since every author adopts an individual method of assessment, there is uncertainty in the interpretation and comparison of different reports. Research on $\mathrm{ONFH}$ and its treatment require a standardised method of quantifying the lesion on MRI. ${ }^{8,9}$

We have analysed several methods and their modifications for accuracy and repeatability and the possibility of their international use.

Y.M. Kim, MD, PhD, Professor

J. H. Ahn, MD, Fellow

H. J. Kim, MD, PhD, Assistant Professor

Department of Orthopaedic Surgery

H. S. Kang, MD, PhD, Associate Professor

Department of Diagnostic Radiology

Seoul National University College of Medicine, 28 Yongondong, Chongnogu, Seoul 110-744, Korea.

Correspondence should be sent to Dr H. J. Kim.

C)1998 British Editorial Society of Bone and Joint Surgery

0301-620X/98/68309 \$2.00

954

\section{Patients and Methods}

We examined 26 hips in 21 patients at an early stage of atraumatic ONFH. There were 18 men and three women with a mean age of 40 years (24 to 59). Between October 1995 and May 1996, all hips were diagnosed and classified using simple radiographs and MRI; six were in Ficat stage I and 20 were in stage II. Aetiological factors included alcoholism in 13 hips and corticosteroid therapy in three. In ten the diagnosis was idiopathic osteonecrosis.

MR imaging. In all patients, MRI was performed using one of three units: a 1.5T Horizon (GE, Milwaukee, Wisconsin), a 1.5T Magnetom SP (Siemens, Erlangen, Germany) or a 1.0T Magnetom Expert (Siemens, Erlangen, Germany). T1-weighted images were obtained in the coronal, sagittal and axial planes, with repetition times (TR) of 440 to $750 \mathrm{~ms}$ and echo times (TE) of 10 to $20 \mathrm{~ms}$ (TR/ $\mathrm{TE}=440$ to $750 / 10$ to 20 ). In all planes, the section thickness was 5 or $6 \mathrm{~mm}$, and there was no gap. The field of view was 25 to $40 \mathrm{~cm}$ and the matrix was (179 to $360) \times(192$ to 512$)$.

Measurement of reference values. Since it is impossible to measure the true volume or true surface area of a necrotic segment, it was presumed that each head image seen on coronal MR slices was a round plate 5 or $6 \mathrm{~mm}$ thick, depending on the section thickness (Fig. 1). For this measurement, MR images were reviewed by the four authors together and all demarcations and reference points were decided by consensus. The outer margin of the low signal intensity band was considered to represent the edge of the necrotic area.

To obtain the reference volume, the area of a necrotic segment and of the whole head on each coronal image was measured using an image analyser (VIDAS Image Processing System; Kontron Bildanayse, Munich, Germany). The following formula was then used to calculate the volume of the necrotic segment as a percentage of the volume of the head:

$\%$ volume of necrotic segment

$$
=\frac{\sum \text { area of necrotic segment }}{\sum \text { area of whole head }} \times 100
$$

To obtain the reference surface area of the necrotic THE JOURNAL OF BONE AND JOINT SURGERY 


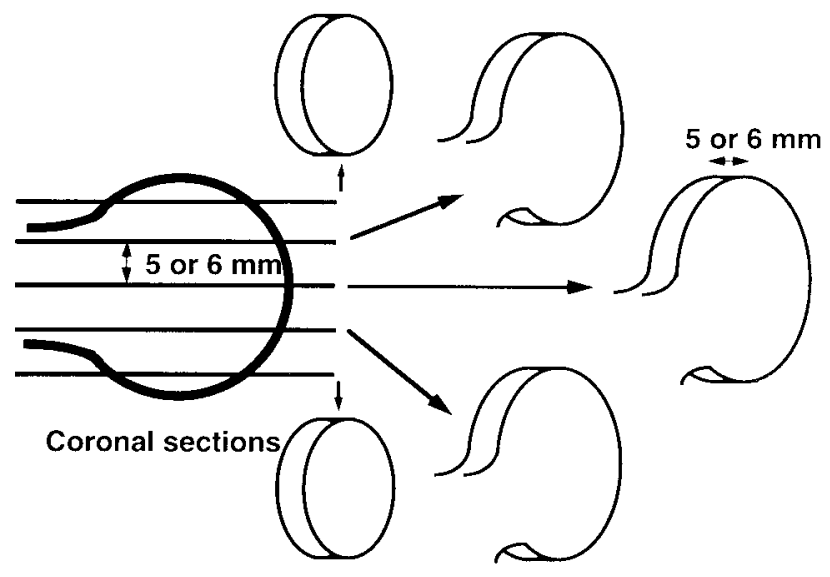

Fig. 1

Diagram showing the measurement of the reference volume and surface area. Each head image, as seen on coronal MR slices, was presumed to be a round plate 5 or $6 \mathrm{~mm}$ thick, depending on the section thickness. segment, the arc of that segment, defined as the necrotic arc angle (NAA), and the diameter of the head were measured on each coronal image. Only the upper half of a head was taken into consideration. The arc of the necrotic segment was expressed by the angle from the concentric centre of each head image (Fig. 2). The following formula was then used to calculate the percentage of the surface area occupied by a necrotic segment:

$\%$ surface area of necrotic segment

$$
=\frac{\Sigma(\text { NAA } \times \text { diameter of head })}{\Sigma(180 \times \text { diameter of head })} \times 100
$$

Estimation of extent of the necrotic lesion using different methods. The extent of a necrotic segment was estimated using the methods of different authors, or their modifications. ${ }^{1-3,8}$ Again the MR images were reviewed by the four authors and all measurements decided by agreement.

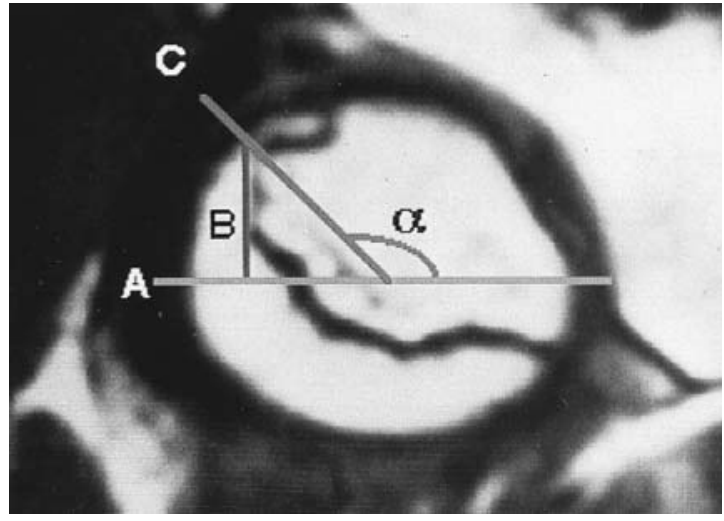

Step 1

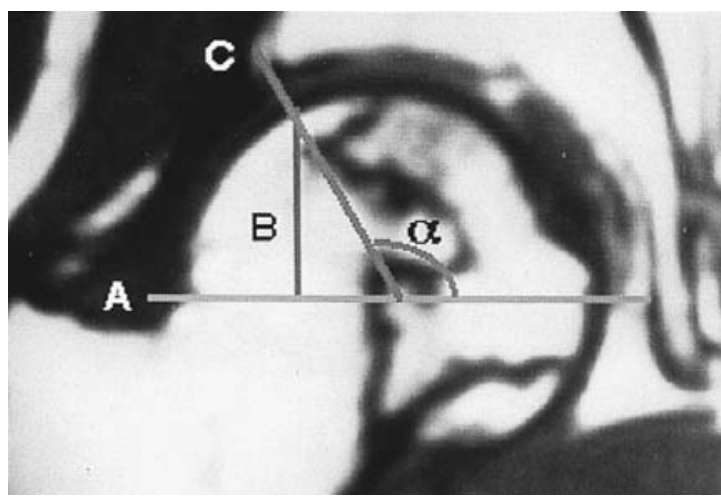

Step 3

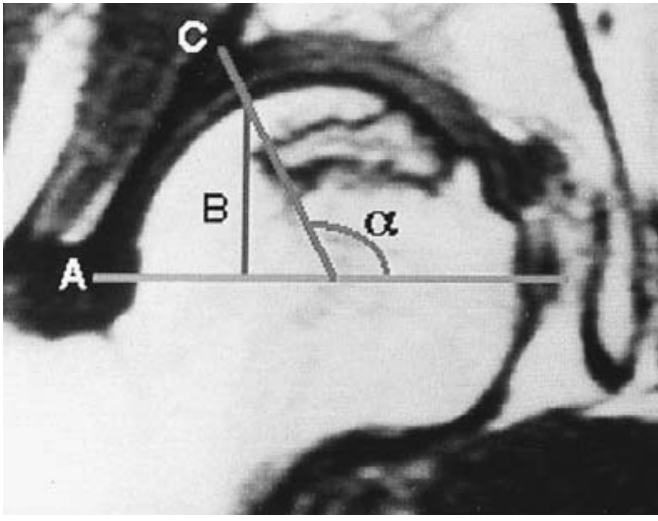

Step 2

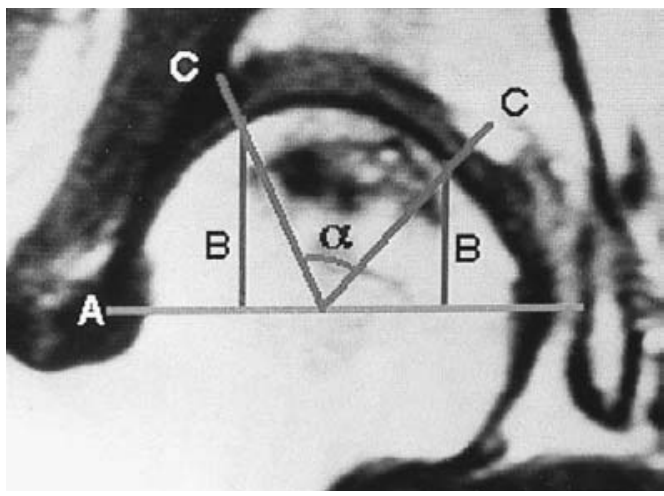

Step 4

Fig. 2

Examples of the measurement of NAA using MRI. Step 1: Using concentric circles, the concentric centre of a head is determined. Step 2: A horizontal line (A) is drawn through the concentric centre. Step 3: From the horizontal line (A), one or two vertical lines (B) are drawn to the medial and lateral ends of the necrotic lesion. Step 4: From the concentric centre, one or two straight lines (C) are drawn to the points where the vertical lines (B) cross the outer margin of a head, and the angle $\alpha$ is measured. 

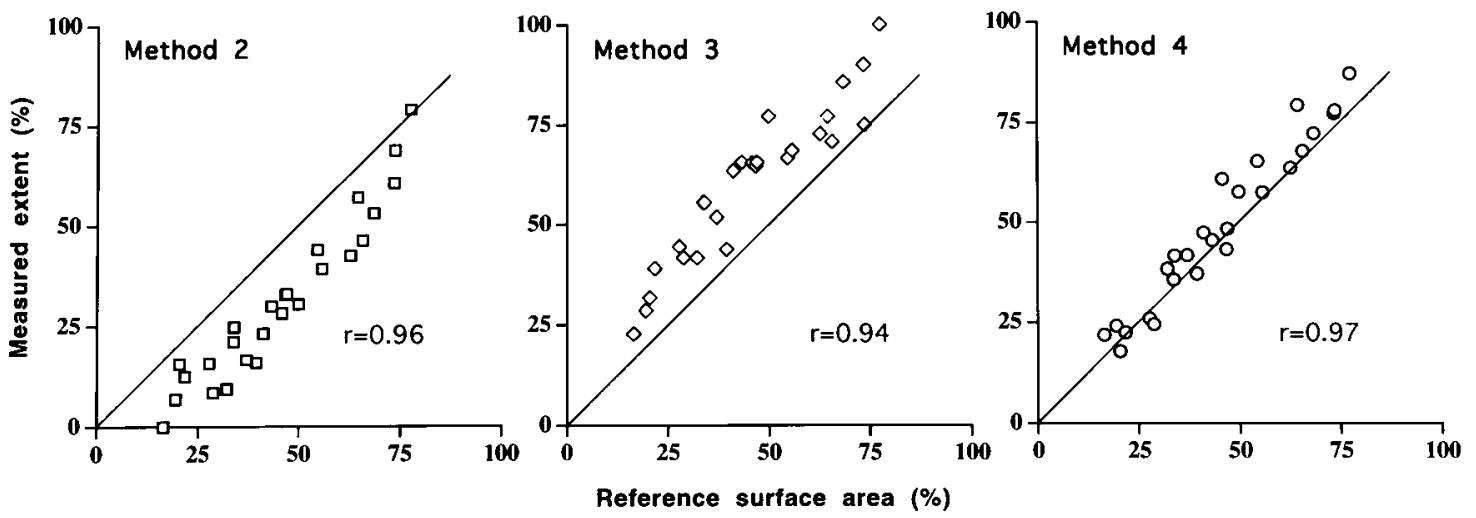

Fig. 3

Scattergrams with line of equality to show the relationship between measured extent using different methods and reference values. Method 2 has a tendency to underestimate the extent and method 3 shows a tendency to overestimate it when the magnitude is high.

Method 1. This is a modification of one of the methods of Lafforgue et al. ${ }^{3}$ The NAA on the midcoronal image (A) was measured and the extent of the necrotic portion was calculated using the formula:

$$
\% \text { of necrotic lesion }=(\mathrm{A} / 180) \times 100 .
$$

Method 2. This is the method of Koo and Kim. ${ }^{8}$ The NAA on the midcoronal image (A) and on the midsagittal image (B) was measured and the extent of the necrotic portion was expressed using the formula:

$\%$ of necrotic lesion $=(\mathrm{A} / 180) \times(\mathrm{B} / 180) \times 100$.

Table I. Difference (\%) between the estimated extent and the reference extent of ONFH using the seven different methods

\begin{tabular}{llrrrrrr}
\hline & \multicolumn{7}{l}{ Method } \\
\cline { 2 - 8 } Reference & $\mathbf{1}$ & \multicolumn{1}{c}{$\mathbf{2}$} & \multicolumn{1}{c}{$\mathbf{3}$} & $\mathbf{4}$ & $\mathbf{5}$ & $\mathbf{6}$ & $\mathbf{7}$ \\
\hline Surface area & & & & & & & \\
$\quad$ Mean & 1.2 & -14.2 & 14.7 & 3.8 & 21.4 & 27.4 & 12.2 \\
$\quad$ SD & 8.1 & 5.8 & 6.3 & 4.8 & 15.6 & 12.8 & 15.4 \\
Volume & & & & & & & \\
$\quad$ Mean & 19.6 & 4.2 & 33.1 & 22.2 & 39.8 & 45.8 & 30.6 \\
SD & 11.4 & 9.0 & 9.6 & 9.6 & 16.3 & 15.5 & 17.6 \\
\hline
\end{tabular}

Method 3. This is the method adopted by the members of our department. On coronal and sagittal images, the total number of images that showed the head (A) and the total number of images that showed the necrotic lesion (B) were counted. The extent of the necrotic lesion was then expressed by the formula:

$\%$ of necrotic lesion $=(\mathrm{B}$ of coronal sections $/$ A of coronal sections $) \times(B$ of sagittal sections/ A of sagittal sections) $\times 100$.

Method 4. The largest anteroposterior diameter of the head $(\mathrm{R})$, the longest anteroposterior length of the necrotic lesion (A) and the longest mediolateral length of the necrotic lesion (B) were measured on axial images. The extent of the necrotic portion was expressed by the equation:

$$
\% \text { of necrotic lesion }=\left\{(\mathrm{A} \times \mathrm{B}) / \mathrm{R}^{2}\right\} \times 100 .
$$

Method 5. This is a method developed by Beltran et al. ${ }^{1}$ The NAA (A) on the coronal image showing the largest necrotic area was measured and the extent of the necrotic portion was expressed by the equation:

$$
\% \text { of necrotic lesion }=(\mathrm{A} / 180) \times 100 \text {. }
$$

\begin{tabular}{|c|c|c|c|c|}
\hline & \multicolumn{4}{|c|}{ Variance (Coefficient of repeatability)* } \\
\hline & \multicolumn{2}{|l|}{ Ten observers } & \multicolumn{2}{|c|}{ Four experienced observers } \\
\hline & Intrapersonal $\dagger$ & Interpersonal $\ddagger$ & Intrapersonal§ & Interpersonal \\
\hline Method 2 & $56.72(15.06)$ & $82.59(18.18)$ & $14.45(7.60)$ & $50.62(14.23)$ \\
\hline Method 3 & $20.00(8.94)$ & $52.54(14.50)$ & $6.23(4.99)$ & $28.21(10.62)$ \\
\hline Method 4 & $25.15(10.02)$ & $38.44(12.40)$ & $4.89(4.42)$ & $18.03(8.49)$ \\
\hline
\end{tabular}

Table II. Repeatability of methods used to determine the extent of osteonecrosis of the femoral head 
Method 6. This is also a method developed by Beltran et al. ${ }^{1}$ The anteroposterior diameter of the head (R) and the longest anteroposterior length of the necrotic lesion (A) were measured on the axial image showing the largest necrotic area. The extent of the necrotic portion was expressed by the formula:

$$
\% \text { of necrotic lesion }=(\mathrm{A} / \mathrm{R}) \times 100 .
$$

Method 7. This is a modification of method 6. The anteroposterior diameter of the head (R) and the longest anteroposterior length of the necrotic lesion (A) were measured on the midaxial image. The extent of the necrotic portion was expressed by the equation:

$$
\% \text { of necrotic lesion }=(\mathrm{A} / \mathrm{R}) \times 100 \text {. }
$$

Agreement between reference values and estimated values. To evaluate the accuracy of each method of estimation, agreement between the reference value and the estimated value was analysed by calculating the mean difference and the standard deviation of the differences. ${ }^{10}$ Since the estimated values can be adjusted for the mean difference, only the standard deviation was taken into consideration. When the standard deviation was less than $5 \%$, the degree of agreement was defined as good; when it was less than $7.5 \%$ it was judged acceptable.

Reproducibility. For the evaluation of the reproducibility of the estimation methods, ten independent observers estimated the extent of each necrotic lesion in six randomly selected hips. Each observer measured each hip six times, with an interval of one week between each measurement. Only estimation methods which demonstrated good or acceptable accuracy, namely methods 2, 3 and 4, were used. Of the ten observers, four were experienced (the present authors) and six were senior residents of the orthopaedic department. The acquired data were analysed for intra- and interpersonal variances and the coefficient of repeatability was calculated; ${ }^{10}$ when this was less than $5 \%$, the reproducibility of a method was considered reasonable.

\section{Results}

Agreement between reference and estimated values. Three of the seven methods demonstrated good (method 4) or acceptable (methods 2 and 3 ) agreement between estimated and reference values of the surface area (Table I, Fig. 3).

Reproducibility. Table II shows the intra- and interpersonal variances of repeated measurement by all ten observers and the four experienced observers using methods 2, 3 and 4 . No method demonstrated reasonable reproducibility for general observers. The repeatability of methods 3 and 4 was reasonable only when they were used by experienced observers.

\section{Discussion}

It is practically impossible to measure the true volume or surface area of a necrotic lesion. We therefore used MR images to calculate the volume and surface area. Holman et $\mathrm{al}^{6}$ and Steinberg et $\mathrm{al}^{9}$ proposed a similar method for the measurement of the extent of the necrotic areas. We believe that these calculated values accurately represent the true values but at the present time this method is too complicated for clinical application.

A number of studies ${ }^{1,3,8}$ have measured the NAA but none has described in detail the method by which this was achieved. In our review, NAA was measured as in Figure 1 because of the extreme irregularity in the shape of the lesions. This is applicable to any irregular shape, but we were not able to apply the method of Kopecky et $\mathrm{al}^{2}$ who estimated the necrotic area by calculating the area of an ellipsoid.

Three of the seven methods demonstrated good or acceptable accuracy, although their repeatability was not satisfactory for clinical use. For two of the three, repeatability was reasonable only when experienced observers were involved. The main cause of variation in method 2 was inconsistency in determination of the midcoronal and midsagittal images. Often, two adjacent head images had almost the same diameter but showed necrotic lesions of very different sizes. Koo and $\mathrm{Kim}^{8}$ reported a coefficient of variation of $3.2 \%$ for the interpersonal variation in method 2. They used magnified instead of conventional images, which suggests that the repeatability of method 2 can be greatly improved by the use of a magnified image.

None of the methods assessed in this study seems appropriate for universal use. Methods 3 and 4 show acceptable accuracy and reasonable repeatability if experienced observers are involved, while the repeatability of method 2 would be improved with magnified MR images. We believe that the study of these three methods will lead to the establishment of personal and institutional criteria for measuring the dimensions of a necrotic lesion in the femoral head.

This study was supported by the Korean Human Technology Research Foundation (KOHTERF-96-02). The authors wish to thank Professor Sangho Lee of the Department of Statistics, Kangwon National University, for his help with the statistical analysis of the data.

No benefits in any form have been received or will be received from a commercial party related directly or indirectly to the subject of this article.

\section{References}

1. Beltran J, Knight CT, Zuelzer WA, et al. Core decompression for avascular necrosis of the femoral head: correlation between longterm results and preoperative MR staging. Radiology 1990;175:533-6.

2. Kopecky KK, Braunstein EM, Brandt KD, et al. Apparent avascular necrosis of the hip: appearance and spontaneous resolution of MR findings in renal allograft recipients. Radiology 1991;179:523-7.

3. Lafforgue P, Dahan E, Chagnaud C, et al. Early-stage avascular necrosis of the femoral head: MR imaging for prognosis in 31 cases with at least 2 years of follow-up. Radiology 1993;187:199-204.

4. Takatori Y, Kokubo T, Ninomiya S, et al. Avascular necrosis of the femoral head: natural history and magnetic resonance imaging. $J$ Bone Joint Surg [Br] 1993;75-B:217-21. 
5. Shimizu K, Moriya H, Akita T, Sakamoto M, Suguro T. Prediction of collapse with magnetic resonance imaging of avascular necrosis of the femoral head. J Bone Joint Surg [Am] 1994;76-A:215-23.

6. Holman AJ, Gardner GC, Richardson ML, Simkin PA. Quantitative magnetic resonance imaging predicts clinical outcome of core decompression for osteonecrosis of the femoral head. J Rheumatol 1995;22:1929-33.

7. Koo K-H, Kim R, Ko G-H, et al. Preventing collapse in early osteonecrosis of the femoral head: a randomised clinical trial of core decompression. J Bone Joint Surg [Br] 1995;77-B:870-4.
8. Koo K-H, Kim R. Quantifying the extent of osteonecrosis of the femoral head: a new method using MRI. J Bone Joint Surg [Br] 1995;77-B:875-80.

9. Steinberg ME, Hayken GD, Steinberg DR. A quantitative system for staging avascular necrosis. J Bone Joint Surg [Br] 1995;77-B: 34-41.

10. Bland JM, Altman DG. Statistical methods for assessing agreement between two methods of clinical measurement. Lancet 1986;i: $307-10$. 